\title{
A KINETIC APPROACH TO THE STUDY OF OPINION FORMATION
}

\author{
LAUREnt Boudin $^{1}$ And Francesco SAlvarani ${ }^{2}$
}

\begin{abstract}
In this work, we use the methods of nonequilibrium statistical mechanics in order to derive an equation which models some mechanisms of opinion formation. After proving the main mathematical properties of the model, we provide some numerical results.
\end{abstract}

Mathematics Subject Classification. 91D10, 82C22.

Received February 15, 2008. Revised October 15, 2008.

Published online February 7, 2009.

\section{INTRODUCTION}

The methods of nonequilibrium statistical mechanics, classically used in the framework of the kinetic theory of gases, can also be fruitfully applied to all the contexts that study the collective behaviour of a large enough number of individuals, where none of which has a preponderant role with respect to the others. For example, kinetic-type equations have been introduced in order to describe a simple market economy with a constant growth mechanism $[5,6,16]$, showing the formation of steady states with (overpopulated) Pareto tails.

Such an approach also seems to be fitted to sociophysics. The main goal of this discipline consists in giving a statistical physics modelling of large scale social phenomena, like opinion formation, cultural dissemination or crowd behaviour [14].

In this paper, we focus on a study of the opinion dynamics in a closed community. The modelling of this phenomenon has already been the subject of numerous works, mainly for its application in politics, to predict the behaviour of voters during an election process or the public opinion tendencies $[8,10,11,13]$.

Several approaches to the problem are possible. For example, kinetic-based models have been proposed in [19] (see also [1]). Moreover, there is a wide literature based on Ising models, introduced in social and political sciences by Galam et al. $[12,14]$. Such a point of view has then been adopted in several papers, for example, $[17,18]$.

In the present work, we aim to describe the time evolution of the opinion set of an isolated population about a particular statement by means of a model only depending on two independent variables: time and opinion.

We shall only consider opinions regarding binary questions. Even if there are many cases where the opinion cannot be easily expressed in terms of "yes" or "no", several important situations (e.g. a referendum) require

\footnotetext{
Keywords and phrases. Sociophysics, opinion formation, kinetic theory.

1 UPMC Université Paris 6, UMR 7598, Laboratoire Jacques-Louis Lions, 75005 Paris, France. laurent.boudin@upmc.fr

2 Dipartimento di Matematica, Università Degli Studi di Pavia, Via Ferrata 1, 27100 Pavia, Italy. francesco.salvarani@unipv .it
} 
that, at the end of a process of opinion formation, the individuals should accept or reject, without reserve, a very precise statement.

The opinion of individuals is represented by a one-dimensional real variable between $(-1)$ and $(+1)$. The choice of the closed interval $[-1,1]$ instead of $\mathbb{R}$ means that extreme opinions can actually be reached, and not only asymptotically (we suggest references $[7,15]$ to readers interested in earlier works on bounded confidence continuous opinion dynamics).

The dynamics of our model is given by the competition of two opposite microscopic effects. The first one is the process of self-thinking, which implies a modification of the agents' opinions. The second one is the interaction between two individuals of the population, who exchange their viewpoints and are then influenced by each other, and is treated as a binary collision. We point out that both elementary sociological phenomena taken into account are well accepted in the literature. The idea to introduce a self confident term in opinion dynamics was first introduced in discrete spin models by Galam and Moscovici [12] and then by Galam [9]. In the present paper, the modifications of the agent opinion due to the self-thinking are modelled as a weighted linear diffusion which vanishes on the boundary of the opinion space.

The binary interactions between individuals are defined as an exchange of opinions with respect to their average value before the collision. This is a difference with respect to [19] or [7], where the collision mechanism is defined starting from the pre-collisional opinion of each agent.

As we shall explain in Section 2, both self-thinking and interaction between individuals are governed, in our model, by operators which are defined up to an opinion-dependent term: the Fourier and the attraction functions, respectively. With such a hypothesis, we assume that the changes of opinions are linked to the opinion itself.

The Fourier and the attraction functions really influence the time evolution of the system, as we shall see in the last section of the paper, devoted to the numerical tests. Of course, their functional form cannot be deduced only from purely mathematical principles, but it should rather be identified by means of some "sociological experiment" such as, for example, polls or sociological studies. Indeed, we do not believe that the dynamics of a closed community is independent on the community itself: for example, different educational systems and different social rules can lead to a completely different kind of interaction between individuals.

For this reason, the mathematical constraints that we impose to these two opinion-dependent terms are quite weak, in order to cover many possible sociological styles. This adaptability to different kinds of community is, in our opinion, one of the main advantages of the model.

Nevertheless, the model is not universal but, as any model, is based on some hypotheses which fix its limits of validity. In particular, a description based on statistical mechanics tools is only fruitful when the size of the population is large enough. Moreover, we suppose in our model that the probability of a binary interaction is constant. In a structured society, this is not true, since people are normally involved in a social network. This means that some meetings are very probable and other ones almost impossible. However, the influence of the network cannot be explained as a function of opinion and time. Since we limit ourselves to consider only these two independent variables, it seems logical to treat, in a first approximation, the probability of a binary interaction as a constant.

All the previous hypotheses have a double effect. On the one hand, they reduce the applicability of the model, on the other hand, by simplifying the phenomena taken into account, they allow to build a model which is tractable from a mathematical point of view.

Of course, a next step in the modelling would be the coupling of our model with other equations which would take into account the existence of social networks and allow more independent variables.

Finally, it is important to point out the motivation of this work, which mixes modelling, analysis, numerical simulations and sociological considerations. Our aim is to provide a tool which shows what kinetic theory can say in a world seemingly as distant from mathematics as sociological dynamics. In this spirit, we have introduced a model which explains - at a qualitative level - several possible behaviours of opinion dynamics in the case of a binary question. 
We do not claim that this model gives the exact reproduction of the reality, since the sociological behaviour of a community is not a physical system. However, it allows to treat, from a mathematical point of view, some important phenomena which come from collective dynamics.

This paper is organized as follows. We first describe our model, including the processes of binary opinion exchanges and self-thinking. Then we prove an existence result for the considered problem. Eventually, we present some numerical tests.

\section{THE MODEL}

In the following, $\Omega$ denotes the open interval $(-1,1)$. We label with $x=-1$ and $x=1$ the two extreme answers to the statement, i.e. "yes" or "no" without reserve, and describe the opinion by the continuous variable $x \in \bar{\Omega}$. Note that any intermediate value between the two extremes (zero excluded) means that the corresponding individual partially agrees with the opinion labeled with the same sign, with a degree of conviction which is proportional to $|x|$. If $x=0$, the corresponding individual has no preference with respect to the question.

The unknown of our model is the density (or distribution function) $f=f(t, x)$, defined on $\mathbb{R}_{+} \times \bar{\Omega}$, whose time evolution is described, as shown later, by an integro-differential equation. The precise meaning of $f$ is the following. Once individuated the population to study, if the opinions are defined on a sub-domain $D \subseteq \bar{\Omega}$, the integral

$$
\int_{D} f(t, x) \mathrm{d} x
$$

represents the number of individuals with opinion included in $D$ at time $t \geq 0$. Note that, in order to give a sense to the previous considerations, it would be adequate if $f$ satisfies $f(t, \cdot) \in L^{1}(\Omega)$ for all $t \in \mathbb{R}_{+}$.

As sketched in the introduction, we shall take into account only two processes of opinion formation. The first one is given by the binary interaction between agents, which exchange their points of view and adjust their opinions on the ground of each other's belief, whereas the second behaviour we take into account is the self-thinking process.

\subsection{Exchange of opinions inside the population}

We model this process by borrowing the collisional mechanism of a typical interaction in the kinetic theory of gases: whereas in rarefied gas dynamics the particles exchange momentum and energy in such a way that the principles of classical mechanics are satisfied, here the "collision" between individuals allows the exchange of opinions.

Let $x, x_{*} \in \bar{\Omega}$ the opinions of two agents before an interaction. We suppose that the opinions after the interaction change according to the following rule. Once found the average opinion before the interaction, each of the two individuals will approach it in a way which guarantees that stronger opinions are less attracted towards the average than weaker opinions through the following formula:

$$
\left\{\begin{array}{l}
x^{\prime}=\frac{x+x_{*}}{2}+\eta(x) \frac{x-x_{*}}{2}, \\
x_{*}^{\prime}=\frac{x+x_{*}}{2}+\eta\left(x_{*}\right) \frac{x_{*}-x}{2} .
\end{array}\right.
$$

The term $\eta: \bar{\Omega} \rightarrow \mathbb{R}$, which we henceforth name the attraction function, is a smooth function which describes the degree of attraction of the average opinion with respect to the starting opinion of the agent. In order to make the model unaffected by the change of label of the two extreme opinions, we shall always suppose that $\eta$ is even. In the sequel, we need some more assumptions on the attraction function $\eta$. 
Definition 2.1. Let $\eta: \bar{\Omega} \rightarrow \mathbb{R}$ be an even function of class $C^{1}(\bar{\Omega})$. The attraction function is admissible if $0 \leq \eta<1, \eta^{\prime}(x)>0$ for all $x>0$, and the Jacobian of the collision mechanism (2.1)

$$
\begin{aligned}
J\left(x, x_{*}\right)= & \frac{1}{2}\left[\eta(x)+\eta\left(x_{*}\right)\right]+\frac{1}{4}\left[\eta^{\prime}(x)-\eta^{\prime}\left(x_{*}\right)\right]\left(x-x_{*}\right) \\
& +\frac{1}{4}\left[\eta^{\prime}(x) \eta\left(x_{*}\right)-\eta(x) \eta^{\prime}\left(x_{*}\right)\right]\left(x-x_{*}\right)-\frac{1}{4} \eta^{\prime}(x) \eta^{\prime}\left(x_{*}\right)\left(x-x_{*}\right)^{2}
\end{aligned}
$$

is uniformly lower bounded by a strictly positive constant, i.e. there exists $\xi>0$ such that $J\left(x, x_{*}\right) \geq \xi$, for any $x, x_{*} \in \bar{\Omega}$.

The first property of $\eta$ avoids that the interaction destroys the bounds of the interval $\Omega$. The second one translates the assumption that stronger opinions are less attracted towards the average than weaker ones, and is well accepted in the literature [19] (see also [7,9]). The third one ensures that the inverse of the collision rule (2.1) is well defined.

Note that the collision mechanism (2.1) is different from the mechanism proposed by Toscani in [19], since here the post-collisional opinions are defined from the mean value of the two pre-collisional opinions. Moreover, it differs from the standard collisional rules of dissipative kinetic models, which are usually linear.

Remark 2.2. By using the properties listed in Definition 2.1, it is not difficult to also prove that, for any $x$, $x_{*} \in \bar{\Omega}$,

and, since $0 \leq \eta<1$,

$$
x^{\prime}-x_{*}^{\prime}=\frac{1}{2}\left(\eta(x)+\eta\left(x_{*}\right)\right)\left(x-x_{*}\right),
$$

$$
\left|x^{\prime}-x_{*}^{\prime}\right|<\left|x-x_{*}\right| .
$$

It is then clear that the lateral bounds are not violated, i.e.

$$
\max \left\{\left|x^{\prime}\right|,\left|x_{*}^{\prime}\right|\right\}<\max \left\{|x|,\left|x_{*}\right|\right\} \cdot
$$

We note that the set of admissible attraction functions is not empty. A possible choice of $\eta$ is the following:

$$
\eta(x)=\lambda\left(1+x^{2}\right), \quad 0<\lambda<1 / 2 .
$$

It is easy to see, when $\eta$ has the form (2.2), that the properties listed in Definition 2.1 are satisfied. The first and the second requirements in Definition 2.1 are obviously fulfilled. Easy but tedious computations finally show that the Jacobian $J$ of the collision rule (2.1), with $\eta$ defined by (2.2), is given by

$$
\begin{aligned}
J\left(x, x_{*}\right)= & \frac{\lambda}{2}\left(2+x^{2}+x_{*}{ }^{2}\right)+\frac{\lambda}{2}\left(x-x_{*}\right)^{2} \\
& +\frac{\lambda^{2}}{2}\left[x\left(1+x_{*}{ }^{2}\right)-\left(1+x^{2}\right) x_{*}\right]\left(x-x_{*}\right)-\lambda^{2} x x_{*}\left(x-x_{*}\right)^{2},
\end{aligned}
$$

and satisfies

$$
J \geq \lambda .
$$

Remark 2.3. The transformation of type (2.1) can be locally dissipative. As a matter of fact, if $\eta$ is, for example, defined by $(2.2)$, with $\lambda \in(0,(\sqrt{2}-1) / 2]$, we have

$$
\begin{aligned}
x^{\prime 2}+x_{*}^{\prime 2}= & x^{2}+x_{*}{ }^{2} \\
& +\frac{\left(x-x_{*}\right)^{2}}{4}\left[\lambda^{2}\left(1+x^{2}\right)^{2}+\lambda^{2}\left(1+x_{*}{ }^{2}\right)^{2}+2 \lambda\left(x+x_{*}\right)^{2}-2\right] \\
\leq & x^{2}+x_{*}{ }^{2}+\frac{1}{2}\left(4 \lambda^{2}+4 \lambda-1\right)\left(x-x_{*}\right)^{2} \\
\leq & x^{2}+x_{*}{ }^{2} .
\end{aligned}
$$


Once defined the collision rule (2.1), the interaction between individuals and the corresponding exchange of opinions is described by a collisional integral of Boltzmann type.

The collisional integral, which will be henceforth denoted as $Q$, has the classical structure of the dissipative Boltzmann kernels. It can be viewed as composed of two parts: a gain term $Q^{+}$, which quantifies the exchanges of opinion between individuals which give, after the interaction with another individual, the opinion $x$, and a loss term $Q^{-}$, which quantifies the exchanges of opinion where an individual with pre-collisional opinion $x$ experiences an interaction with another member of the population.

It is apparent that, in general, the existence of a pre-collisional pair which returns the post-collisional pair $\left(x, x_{*}\right)$ through a collision of type (2.1) is not guaranteed, unless we suppose that the collisional rule is a diffeomorphism of $\bar{\Omega}^{2}$ onto itself. Unfortunately, the collisional mechanism (2.1) does not verify this property. For instance, there is no $\left(x, x_{*}\right) \in \bar{\Omega}^{2}$ which gives, after collision, the couple of extreme opinions $(-1,1)$.

In order to overcome this difficulty, the natural framework for such a collision rule is given by the weak form. Two choices are possible. We may either build a model in a weak form with respect to $x$ only, or to work in a weak setting with respect to the whole set of independent variables. We choose the first option, which seems to be the correct framework for such kind of models.

Let $\phi=\phi(x)$ be a suitably regular test function. We define the weak form of the collision kernel as

$$
\langle Q(f, f), \phi\rangle=\beta \iint_{\Omega^{2}} f(t, x) f\left(t, x_{*}\right)\left[\phi\left(x^{\prime}\right)-\phi(x)\right] \mathrm{d} x_{*} \mathrm{~d} x .
$$

Note that the particular form of the collision rule (2.1) only enters through the test function $\phi\left(x^{\prime}\right)$. The cross section $\beta>0$ is a parameter which governs the probability that an exchange of opinions can occur. In our model, we suppose that $\beta$ is purely constant. This is the simplest possible assumption, which means that the probability of interaction of two individuals does not depend on their respective opinions. Of course, other choices, based on sociological considerations, are possible.

It is also clear that the operator $Q$ only acts on the variable of opinion, and not on time.

The explicit form of the change of variables (2.1) also allows to give the following alternative formulations of the collision kernel:

$$
\begin{aligned}
\langle Q(f, f), \phi\rangle & =\beta \iint_{\Omega^{2}} f(t, x) f\left(t, x_{*}\right)\left[\phi\left(x_{*}^{\prime}\right)-\phi\left(x_{*}\right)\right] \mathrm{d} x_{*} \mathrm{~d} x \\
& =\frac{\beta}{2} \iint_{\Omega^{2}} f(t, x) f\left(t, x_{*}\right)\left[\phi\left(x^{\prime}\right)+\phi\left(x_{*}^{\prime}\right)-\phi(x)-\phi\left(x_{*}\right)\right] \mathrm{d} x_{*} \mathrm{~d} x .
\end{aligned}
$$

Remark 2.4. At least formally, we have $\langle Q(f, f), 1\rangle=0$.

The form of the collisional integral given by (2.3) is nevertheless not completely satisfactory for the gain term because of the intricate dependence of the argument of the test function with respect to the variables $x, x_{*}$. We therefore consider the weak form of the gain term

$$
\left\langle Q^{+}(f, f), \phi\right\rangle=\beta \iint_{\Omega^{2}} f(t, x) f\left(t, x_{*}\right) \phi\left(x^{\prime}\right) \mathrm{d} x_{*} \mathrm{~d} x .
$$

Let us denote

$$
D_{\eta}=\left\{\left(x, x^{\prime}\right) \in \mathbb{R} \times \bar{\Omega} \mid \frac{x^{\prime}-1}{2}+\eta\left(x^{\prime}\right) \frac{x^{\prime}+1}{2} \leq x \leq \frac{x^{\prime}+1}{2}+\eta\left(x^{\prime}\right) \frac{x^{\prime}-1}{2}\right\}
$$

and

$$
K_{\eta}\left(x, x^{\prime}\right)=\frac{2 \beta}{1-\eta\left(x^{\prime}\right)} \chi_{D_{\eta}}\left(x, x^{\prime}\right), \quad \forall x, x^{\prime} \in \bar{\Omega},
$$


where $\chi_{D_{\eta}}$ is the characteristic function of the set $D_{\eta}$. Since $\eta$ is an admissible attraction function, it is clear that $D_{\eta} \subseteq \bar{\Omega}^{2}$.

We then perform the change of variable $x_{*} \mapsto x^{\prime}$ in (2.4), for a fixed $x$. It is easy to see that

$$
\mathrm{d} x_{*}=\frac{2}{1-\eta(x)} \mathrm{d} x^{\prime} \quad \text { and } \quad x_{*}=\frac{2 x^{\prime}-x-\eta(x) x}{1-\eta(x)} .
$$

Then, after permuting $x$ and $x^{\prime}$, we obtain the following weak form of the gain term:

$$
\left\langle Q^{+}(f, f), \phi\right\rangle=\iint_{\Omega^{2}} K_{\eta}\left(x, x^{\prime}\right) f\left(t, \frac{2 x-x^{\prime}-\eta\left(x^{\prime}\right) x^{\prime}}{1-\eta\left(x^{\prime}\right)}\right) f\left(t, x^{\prime}\right) \phi(x) \mathrm{d} x^{\prime} \mathrm{d} x .
$$

A new weak form of the collision operator immediately comes:

$$
\langle Q(f, f), \phi\rangle=\left\langle Q^{+}(f, f), \phi\right\rangle-\beta \iint_{\Omega^{2}} f(t, x) f\left(t, x^{\prime}\right) \phi(x) \mathrm{d} x^{\prime} \mathrm{d} x,
$$

which will be henceforth our definition of the collisional kernel for our model.

It is then straightforward to prove the following lemma.

Lemma 2.5. Let $f(t, \cdot) \in L^{1}(\Omega)$. Then both $Q^{+}(f, f)(t, \cdot)$ and $Q(f, f)(t, \cdot)$ are of class $L^{1}(\Omega)$, and we have, for a.e. $t$,

$$
\begin{aligned}
\left\|Q^{+}(f, f)(t, \cdot)\right\|_{L^{1}(\Omega)} & \leq \frac{2 \beta}{1-\max \eta}\|f(t, \cdot)\|_{L^{1}(\Omega)}^{2}, \\
\|Q(f, f)(t, \cdot)\|_{L^{1}(\Omega)} & \leq\left(\frac{2}{1-\max \eta}+1\right) \beta\|f(t, \cdot)\|_{L^{1}(\Omega)}^{2} .
\end{aligned}
$$

\subsection{Self-thinking}

As already mentioned in Section 1, the self-thinking phenomenon is described, in our model, by a diffusion operator obeying to a non-uniform Fourier law, with Fourier term $\alpha=\alpha(x)$. This means that we introduce a term which has the structure $\left(\alpha f_{x}\right)_{x}$, which will compete with the collision operator describing the interactions between the agents.

It is well known, indeed, that diffusion is an essential element of opinion dynamics, since it quantifies the possibility that people may change their opinion either on their own or due to interaction with media [2].

In our case, the diffusion term is weighted by a non homogeneous quantity which satisfies the assumptions listed in the following definition:

Definition 2.6. Let $\alpha: \bar{\Omega} \rightarrow \mathbb{R}$ be a nonnegative function of class $C^{1}(\bar{\Omega})$. We say that $\alpha$ is admissible if $\alpha(x)=\alpha(-x)$ for all $x \in \bar{\Omega}$ and $\alpha(-1)=\alpha(1)=0$.

The first property (symmetry) allows to treat in an equivalent way positive and negative opinions. The second one forces the diffusive process to respect the bounds of the opinion space $\bar{\Omega}$.

Remark 2.7. Note that $\left(\alpha f_{x}\right)_{x}$ has a meaning in the distributional sense if $f(t, \cdot) \in L^{1}(\Omega)$. Indeed, since $\alpha \in W^{1, \infty}(\Omega)$ (in fact $C^{1}(\bar{\Omega})$ ), we can write, in the distributional sense:

$$
\left(\alpha f_{x}\right)_{x}=(\alpha f)_{x x}-\left(f \alpha_{x}\right)_{x} .
$$

A possible choice of $\alpha$ is, for example, $\alpha(x)=\kappa\left(1-x^{2}\right)$, with $\kappa>0$. This profile translates the idea that individuals with a stronger opinion are more stable in their convictions. As in the case of the attraction function, other choices, based on psychological studies, are obviously possible. 


\subsection{Combining the two phenomena}

We are then able to write down the whole model, by considering both the interaction effect and the selfthinking. The evolution law of the unknown $f=f(t, x)$ results in a partial integro-differential equation of second order with respect to the opinion variable:

$$
\int_{\Omega} f_{t}(t, x) \varphi(x) \mathrm{d} x=\int_{\Omega}\left[\alpha(x) \varphi_{x}(x)\right]_{x} f(t, x) \mathrm{d} x+\langle Q(f, f), \varphi\rangle
$$

posed in $(t, x) \in[0, T] \times \Omega, T>0$, for all $\varphi \in C^{2}(\bar{\Omega})$, with initial condition

$$
f(0, x)=f^{\text {in }}(x) \text { for all } x \in \bar{\Omega} .
$$

\section{Main mathematical PROPERTies}

This section is devoted to state and study some mathematical properties of equations (2.8)-(2.9). We first obtain some a priori estimates and then deduce a theorem which proves the existence of weak solutions to (2.8)-(2.9).

Our model guarantees the conservation of the total number of individuals of the population. By borrowing the kinetic theory language, the following result is also named the conservation of the total mass.

Proposition 3.1. Let $f=f(t, x)$ be a nonnegative weak solution of (2.8)-(2.9), with a nonnegative initial condition $f^{\text {in }} \in L^{1}(\Omega)$. Then we have

$$
\|f(t, \cdot)\|_{L^{1}(\Omega)}=\left\|f^{\mathrm{in}}\right\|_{L^{1}(\Omega)} \quad \text { for a.e. } t \geq 0 .
$$

Proof. We simply consider equation (2.8) with test function $\varphi \equiv 1$; the thesis immediately follows.

Mass conservation is not realistic if we consider long-time forecasts. Indeed, in such situations, we should also consider processes of birth and death, which would lead to oscillations in the total number of individuals.

But usually, as in the case of elections or referendums, the interest of such models is to deduce short-term forecast by using, as an initial datum, the result of some opinion poll. We also have to point out that the poll is in fact the comparison at a given time $t$ between the two integrals

$$
\int_{\Omega_{-}} f(t, x) \mathrm{d} x \quad \text { and } \quad \int_{\Omega_{+}} f(t, x) \mathrm{d} x,
$$

where $\Omega_{-}=(-1,0)$ and $\Omega_{+}=(0,1)$. That is why we are also interested in computing both integrals in the numerical experiments described in Section 4.

Moreover, since $|x| \leq 1$, from the conservation of mass, we immediately deduce that all the moments of $f$ are bounded.

Corollary 3.2. Let $f=f(t, x)$ be a nonnegative weak solution of problem (2.8)-(2.9), with nonnegative initial condition $f^{\text {in }} \in L^{1}(\Omega)$. Then

$$
\int_{\Omega} x^{n} f(t, x) \mathrm{d} x \leq\left\|f^{\mathrm{in}}\right\|_{L^{1}(\Omega)}, \quad \text { a.e. } t \geq 0
$$

In order to prove the existence of weak solutions of our model, we need the following result.

Proposition 3.3. Consider the initial-boundary value problem for the unknown $v=v(t, x), x \in \Omega$ and $t \in[0, T]$,

$$
v_{t}-\left[\alpha(x) v_{x}\right]_{x}+\mu v=g, \quad \mu \geq 0
$$


with initial condition

$$
v(0, \cdot)=v^{\text {in }}
$$

and boundary conditions

$$
\lim _{x \rightarrow \pm 1} \alpha(x) v_{x}(t, x)=0 \quad \text { a.e. } t,
$$

where $v^{\text {in }} \in L^{1}(\Omega), g \in C\left([0, T] ; L^{1}(\Omega)\right)$ are nonnegative functions. Then (3.1)-(3.3) admits a unique solution $v \in C^{0}\left([0, T] ; L^{1}(\Omega)\right)$, and $v$ is nonnegative.

Proof. Let $u=u(t, x)$ the solution of the initial-boundary value problem

$$
\begin{gathered}
u_{t}-\left[\alpha(x) u_{x}\right]_{x}=0, \\
u(0, x)=v^{\text {in }}(x), \quad \lim _{x \rightarrow \pm 1} \alpha(x) u_{x}(t, x)=0 .
\end{gathered}
$$

The existence and uniqueness theorem for this problem is the main result of [4]. Moreover, in the same paper it is proved that $u$ is nonnegative and belongs to $C^{0}\left([0, T] ; L^{1}(\Omega)\right)$.

We now consider the function $y(t, x)=u(t, x) \exp (-\mu t)$. It is easy to see that $y$ solves equation (3.1) with initial and boundary data (3.2)-(3.3) when $g \equiv 0$.

We now claim that Proposition 3.3 easily follows by using the Duhamel principle. By denoting $w=w(t, x ; s)$, $x \in \Omega, t \in[0, T], s \leq t$, the solution of

$$
w_{t}(t, x ; s)-\left[\alpha(x) w_{x}(t, x ; s)\right]_{x}+\mu w(t, x ; s)=0, \quad \mu \geq 0,
$$

with initial datum $($ at $t=s)$

$$
w(s, x ; s)=g(s, x)
$$

and boundary conditions

$$
\lim _{x \rightarrow \pm 1} \alpha(x) w_{x}(t, x ; s)=0
$$

then

$$
z(t, x)=\int_{0}^{t} w(t, x ; s) \mathrm{d} s
$$

solves equation (3.1), with vanishing initial condition and boundary data given by (3.3).

By linearity, it is now easy to deduce that $v(t, x)=y(t, x)+z(t, x)$ solves equation (3.1) with initial and boundary data $(3.2)-(3.3)$.

The regularity properties for $v$ and the uniqueness of the solution immediately follow.

As pointed out in [4], the singular Neumann boundary conditions (3.3) are the only ones which allow a unified treatment of the initial-boundary value problem for the evolution equation (3.1) independently on the degeneracy of $\alpha$. In particular, they are automatically satisfied if $\alpha$ is highly degenerate and reduce to the classical Wentcel conditions of boundary regularity of the solutions for low order zeros.

Thanks to the previous result, we are able to prove the following existence theorem.

Theorem 3.4. Let $f^{\text {in }}$ a nonnegative function of class $L^{1}(\Omega)$. Then there exists a nonnegative weak solution $f \in L^{\infty}\left(0, T ; L^{1}(\Omega)\right)$ of problem $(2.8)-(2.9)$, that is of

$$
\int_{\Omega} f_{t}(t, x) \varphi(x) \mathrm{d} x=\int_{\Omega}\left[\alpha(x) \varphi_{x}(x)\right]_{x} f(t, x) \mathrm{d} x+\langle Q(f, f), \varphi\rangle,
$$

for all $\varphi \in C^{2}(\bar{\Omega})$, where the equation takes sense in $\mathcal{D}^{\prime}(-T, T)$ with initial condition $f(0, x)=f^{\text {in }}(x)$ for all $x \in \Omega$. 
Proof. Let us set

$$
\rho=\int_{\Omega} f^{\text {in }}\left(x_{*}\right) \mathrm{d} x_{*},
$$

and consider the sequence $\left(f^{n}\right)_{n \in \mathbb{N}}$ defined by finite induction as $f^{0} \equiv 0$ and as solutions of

$$
\int_{\Omega} f_{t}^{n+1} \varphi \mathrm{d} x-\int_{\Omega}\left(\alpha \varphi_{x}\right)_{x} f^{n+1} \mathrm{~d} x+\beta \rho \int_{\Omega} f^{n+1} \varphi \mathrm{d} x=\left\langle Q^{+}\left(f^{n}, f^{n}\right), \varphi\right\rangle,
$$

for all $\varphi \in C^{2}(\bar{\Omega})$, together with the initial conditions

$$
f^{n}(0, \cdot)=f^{\text {in }}, \quad n \geq 1
$$

and the boundary conditions

$$
\lim _{x \rightarrow \pm 1} \alpha(x) f_{x}^{n}(t, x)=0 \quad \text { a.e. } t, \quad n \geq 1 .
$$

Thanks to Lemma 2.5, we can apply Proposition 3.3 and deduce, by induction, that $f^{n}$ exists, belongs to $C^{0}\left([0, T] ; L^{1}(\Omega)\right)$ and is nonnegative.

If we pick $\varphi \equiv 1$ in $(3.4)$, it comes

$$
\frac{\mathrm{d}}{\mathrm{d} t} \int_{\Omega} f^{n+1} \mathrm{~d} x+\beta \rho \int_{\Omega} f^{n+1} \mathrm{~d} x=\beta\left(\int_{\Omega} f^{n} \mathrm{~d} x\right)^{2} .
$$

Therefore, by finite induction, we immediately get

$$
\int_{\Omega} f^{n} \mathrm{~d} x \leq \rho, \quad n \geq 1
$$

Moreover, $\left(f^{n}\right)$ is a non-decreasing sequence. Indeed, for $n \geq 1$, if we subtract equation (3.4) for $f^{n}$ from equation (3.4) for $f^{n+1}$, the quantity $\left(f^{n+1}-f^{n}\right)$ satisfies the following equation, which is of type (3.1):

$$
\begin{aligned}
\int_{\Omega}\left(f^{n+1}-f^{n}\right)_{t} \varphi \mathrm{d} x-\int_{\Omega}\left(\alpha \varphi_{x}\right)_{x}\left(f^{n+1}-f^{n}\right) \mathrm{d} x & = \\
& \left\langle Q^{+}\left(f^{n}, f^{n}\right), \varphi\right\rangle-\left\langle Q^{+}\left(f^{n-1}, f^{n-1}\right), \varphi\right\rangle-\beta \rho \int_{\Omega}\left(f^{n+1}-f^{n}\right) \varphi \mathrm{d} x
\end{aligned}
$$

with initial datum equal to 0 and singular Neumann boundary conditions (3.3). We know that $f^{0} \equiv 0$, and that $f^{1} \geq 0$ by Proposition 3.3. If we suppose, by induction, that $f^{n} \geq f^{n-1}$, then the second member of the previous equation is nonnegative, and hence Proposition 3.3 implies that $\left(f^{n+1}-f^{n}\right) \geq 0$, i.e. $f^{n+1} \geq f^{n}$.

Therefore, by monotone convergence, there exists $f \in L^{\infty}\left(0, T ; L^{1}(\Omega)\right)$ such that $\left(f^{n}\right)$ converges to $f$ almost everywhere and in $L^{\infty}\left(0, T ; L^{1}(\Omega)\right)$.

Equality (2.9) is clear. Let us prove now that $f$ solves $(2.8)$ in $\mathcal{D}_{t}^{\prime}$. First of all, for all $\varphi \in C^{2}(\bar{\Omega})$ and $\psi \in \mathcal{D}(-T, T)$, the quantities

$$
\int_{0}^{T} \int_{\Omega} f^{n+1}(t, x) \varphi(x) \psi(t) \mathrm{d} x \mathrm{~d} t+\int_{\Omega} f^{n+1}(0, x) \varphi(x) \psi(0) \mathrm{d} x
$$

and

$$
\int_{0}^{T} \int_{\Omega}\left[\alpha(x) \varphi_{x}(x)\right]_{x} f^{n+1}(t, x) \psi(t) \mathrm{d} x \mathrm{~d} t
$$


are clearly convergent when $n$ goes to $+\infty$, respectively towards

$$
\int_{0}^{T} \int_{\Omega} f(t, x) \varphi(x) \psi(t) \mathrm{d} x \mathrm{~d} t+\int_{\Omega} f^{\mathrm{in}}(x) \varphi(x) \psi(0) \mathrm{d} x
$$

and

$$
\int_{0}^{T} \int_{\Omega}\left[\alpha(x) \varphi_{x}(x)\right]_{x} f(t, x) \psi(t) \mathrm{d} x \mathrm{~d} t
$$

for all $\varphi \in C^{2}(\bar{\Omega})$ and $\psi \in \mathcal{D}(-T, T)$.

For the collision term, the loss part easily is treated because of mass conservation given by Lemma 3.1

$$
\beta \rho \int_{0}^{T} \int_{\Omega} f^{n+1}(x) \varphi(x) \psi(t) \mathrm{d} x \mathrm{~d} t \rightarrow \beta \int_{0}^{T} \iint_{\Omega^{2}} f\left(x_{*}\right) f(x) \varphi(x) \psi(t) \mathrm{d} x \mathrm{~d} x_{*} \mathrm{~d} t
$$

when $n$ goes to $+\infty$, for all $\varphi \in C^{2}(\bar{\Omega})$ and $\psi \in \mathcal{D}(-T, T)$.

Finally, we are concerned with the nonlinear limit, when $n$ goes to $+\infty$, of

$$
\beta \int_{0}^{T} \iint_{\Omega^{2}} f^{n}(x) f^{n}\left(x_{*}\right) \varphi\left(x^{\prime}\right) \psi(t) \mathrm{d} x \mathrm{~d} x_{*} \mathrm{~d} t,
$$

with $\varphi \in C^{2}(\Omega)$ and $\psi \in \mathcal{D}(-T, T)$. For the sake of simplicity, we assume that $\|\varphi\|_{L^{\infty}(\Omega)}=1$. We successively have

$$
\begin{aligned}
\iint_{\Omega^{2}}\left|f^{n}(x) f^{n}\left(x_{*}\right)-f(x) f\left(x_{*}\right)\right|\left|\varphi\left(x^{\prime}\right)\right| \mathrm{d} x \mathrm{~d} x_{*} \leq & \iint_{\Omega^{2}}\left|f^{n}(x)-f(x)\right| f^{n}\left(x_{*}\right) \mathrm{d} x \mathrm{~d} x_{*} \\
& +\iint_{\Omega^{2}}\left|f^{n}\left(x_{*}\right)-f\left(x_{*}\right)\right| f(x) \mathrm{d} x \mathrm{~d} x_{*} \\
\leq & 2 \rho\left\|f^{n}(t, \cdot)-f(t, \cdot)\right\|_{L^{1}(\Omega)}
\end{aligned}
$$

which obviously goes to 0 when $n$ goes to $+\infty$.

By collecting the previous results, we hence deduce that $f$ satisfies (2.8). The proof is therefore complete.

Remark 3.5. The previous theorem gives an existence proof of the problem (2.8)-(2.9), without uniqueness, and does not provide either a characterization of the solutions in term of boundary data, even if they have been built as limit of solutions of problem (3.1)-(3.3), which satisfy boundary conditions of type (3.3). This happens because we have limited ourselves to build a theory in $L^{1}(\Omega)$, and therefore the meaning of the trace of the solution on the space boundary is not guaranteed.

Of course, if one supposes that $f(t, \cdot)$ belongs to $C^{2}(\bar{\Omega})$, we would automatically have that $\lim \alpha(x) f_{x}^{n}(t, x)=0$ when $x \rightarrow \pm 1$. In the next section, we shall treat the solution as a smooth function of class $C^{1}\left([0, T] ; C^{2}(\bar{\Omega})\right)$, and therefore the selected numerical solution satisfies in a natural way the singular Neumann conditions (3.3).

\section{Numerical TESTS}

In this section, we present some numerical results. The computations were performed using a numerical code written in C. We consider a regular subdivision $\left(x_{0}, \ldots, x_{N}\right)$ of $\Omega$, with $N \geq 1$. The function $f$ is computed at the center $x_{i+1 / 2}$ of each interval $\left[x_{i}, x_{i+1}\right], 0 \leq i \leq N-1$.

The scheme itself conserves the total agents number, i.e. $\|f(t)\|_{L_{x}^{1}}$, and is time-split into a diffusion part and a collision one. The diffusion part is here a standard explicit scheme and the collision one uses a slightly modified Bird method [3]. Since the diffusive scheme is explicit, the diffusion time step can be small with respect to the collision time step, so that we need several diffusion steps for one collision step. 


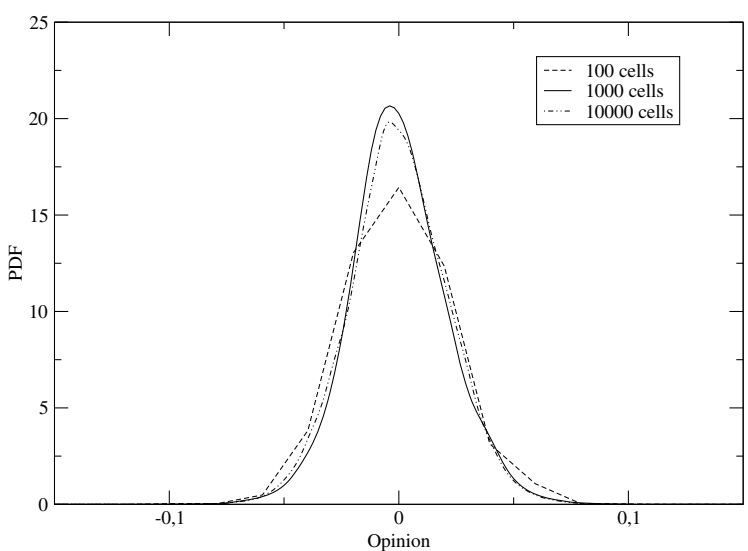

Figure 1. Mesh accuracy.

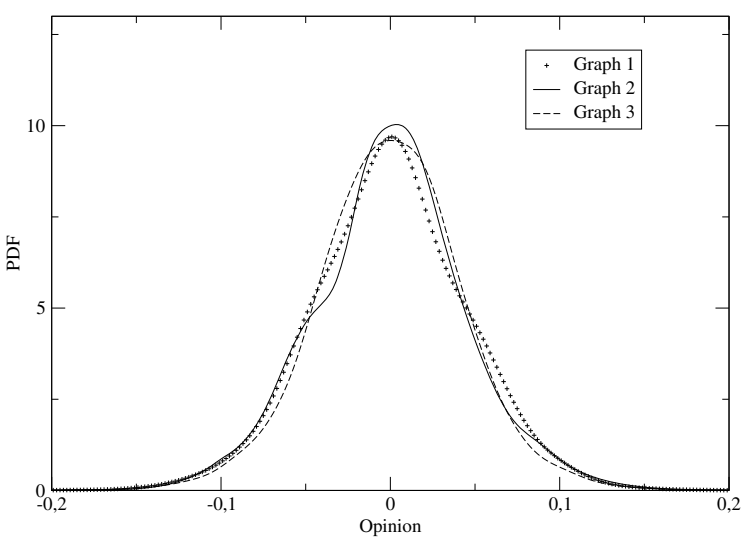

Figure 2. Randomness.

Note that our scheme does not allow the opinions to go out $[-1,1]$. As a matter of fact, opinions $x$ such that $|x|>1$ are not possible because the collision mechanism prevents them, and because the numerical values of the diffusion coefficient $\alpha$ exactly satisfy $\alpha( \pm 1)=0$.

In the following computations, the Fourier term is set under the form $\alpha(x)=\kappa\left(1-x^{2}\right)^{1 / 3}$, which is admissible in the sense of Definition 2.6. The collision frequency is fixed $\beta=50$, and the attraction function is given by $\eta(x)=0.25\left(1+x^{2}\right)$, which is admissible in the sense of Definition 2.1.

As already explained in Section 3, we are also interested in the computations of the integrals of $f$ over $(-1,0)$ and $(0,1)$. They are respectively denoted by $I_{-}(t)$ (number of agents who favour negative opinions) and $I_{+}(t)$ (number of agents who favour positive opinions).

\subsection{Sensitivity with respect to the mesh accuracy}

For this computation, we choose $\kappa=0.001$ and a constant initial datum equal to $1 / 2$, and show the result for final time $T=3$. Figure 1 shows a qualitative difference of computation between $N=100$ meshes and $N=1000$ meshes, whereas the solutions computed for $N=1000$ and $N=10000$ are rather close. Note that the previous statement of course holds for times $T \geq 3$.

From now on, the computations we present are made for $N=1000$. 


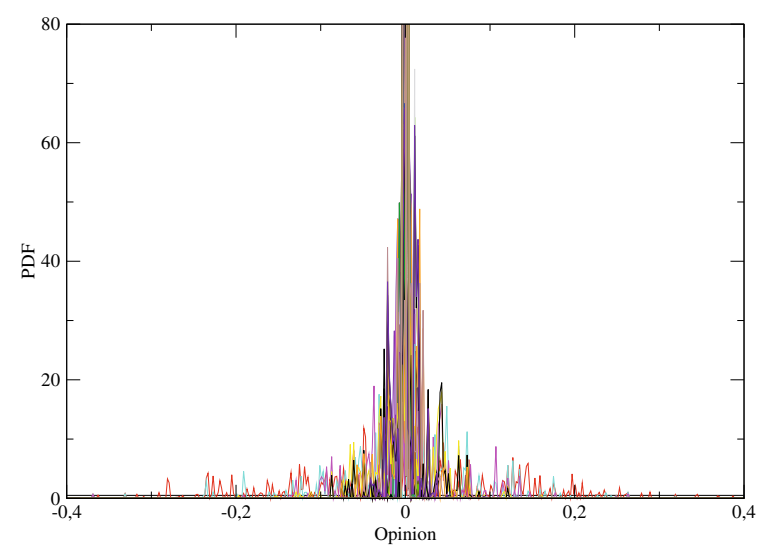

Figure 3. Graphs of $f$ with respect to $t$.

\subsection{Sensitivity with respect to randomness}

The numerical collision process needs one call to the srand function of C. We here choose $\kappa=0.01$ and a uniform initial datum equal to $1 / 2$, and show the result for final time $T=10$. We tried 20 different computations, the corresponding graphs are very close to one of the three graphs presented in Figure 2.

\subsection{Initial uniformly distributed opinions}

We choose a constant initial datum equal to $1 / 2$ again. If there is no diffusion $(\kappa=0)$, the result we obtain (Fig. 3) is really close to Figure 1 in [7]. The distribution function $f$ seems to converge very fast (with respect to time) to a centred Dirac mass in 0. This behaviour fits the model forecast (the collisions have this effect of concentration) and many numerical experiments in the sociophysics literature. In [7], one can also see in some situations the asymptotic formation of two (or more) Dirac masses ("peaks"). Such situations are forbidden by our collision mechanism (2.1).

With the same initial datum, let us set $\kappa=0.05$. The distribution function cannot converge to a Dirac mass anymore but seems to reach quite fast an asymptotic equilibrium (see Fig. 4). We can also note the profile of $f$ is almost centred, but not completely. That also explains why there are oscillations of both $I_{-}$and $I_{+}$: one can check the profile of $I_{+}$in Figure 5. In terms of poll, starting from a uniformly distributed opinion, we can only ensure that number of agents who favour positive (or negative) opinions will approximately vary between $47 \%$ and $53 \%$.

\subsection{Initial monomodal profile}

From now on, we take $\kappa=0.05$. Here we choose a monomodal initial datum. More precisely, from -1 to $-1 / 2, f^{\text {in }}$ equals 2 , and anywhere else equals 0 . The solution $f$ quickly reaches an equilibrium (see Fig. 6). We also note that the support of $f$ is larger than the one of its initial condition and is not centred at -0.75 . Seemingly, the diffusive process moved the center of the profile of $f$. Anyway, at time $t=4,100 \%$ of the individuals still have positive opinions. The profile of $f$ remains very similar for times $t \geq 4$.

\subsection{Two balanced opposite biases case}

Here we deal with a centred bimodal initial datum. More precisely, from -1 to $-1 / 2$, and from $1 / 2$ to $1, f^{\text {in }}$ is set to 1 , and elsewhere equals 0 . Once again, the solution quickly reaches an equilibrium (see Fig. 7). The support of $f$ is then widely modified, since it is a segment almost centred on the middle of the two components of the support of $f^{\text {in }}$, i.e. 0 . The result of the poll is however not very different from the initial datum (see Fig. 8), 


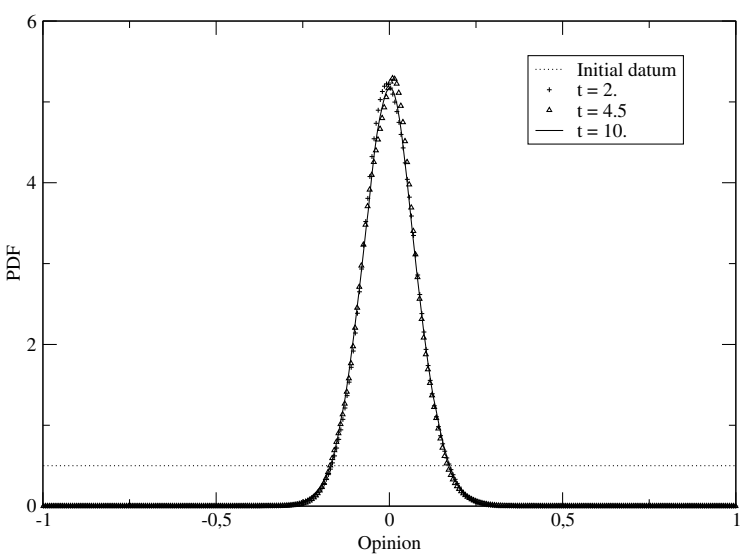

FIgURE 4. Initial uniformly distributed opinion.

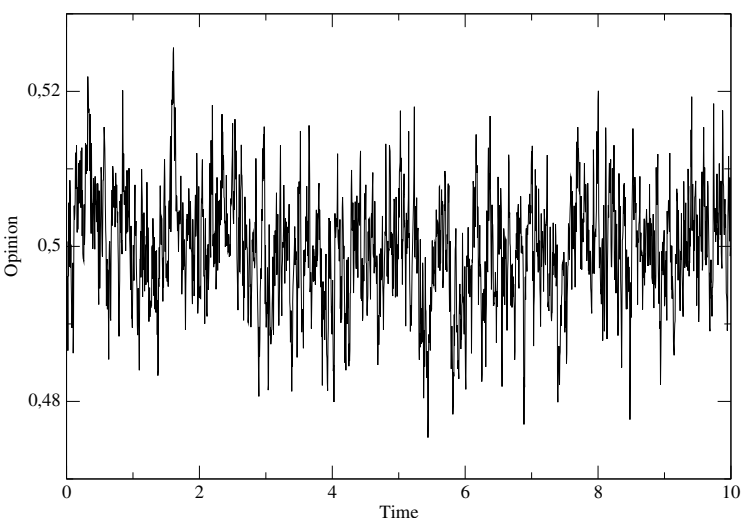

FIGURE 5. Evolution of $I_{+}$with respect to time (constant initial datum).

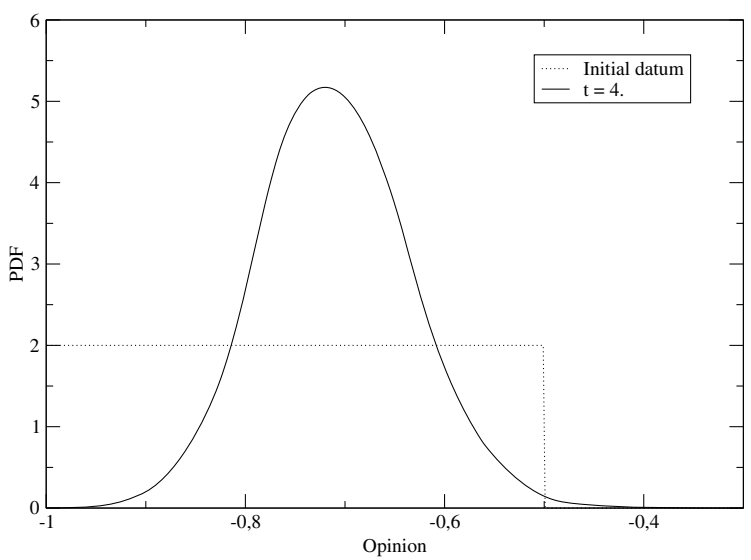

Figure 6. Monomodal initial datum. 


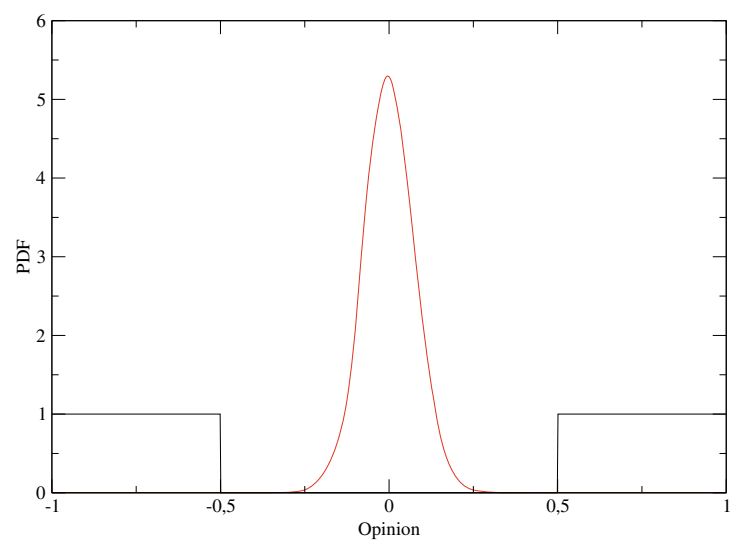

FigurE 7. Balanced opposite biases case.

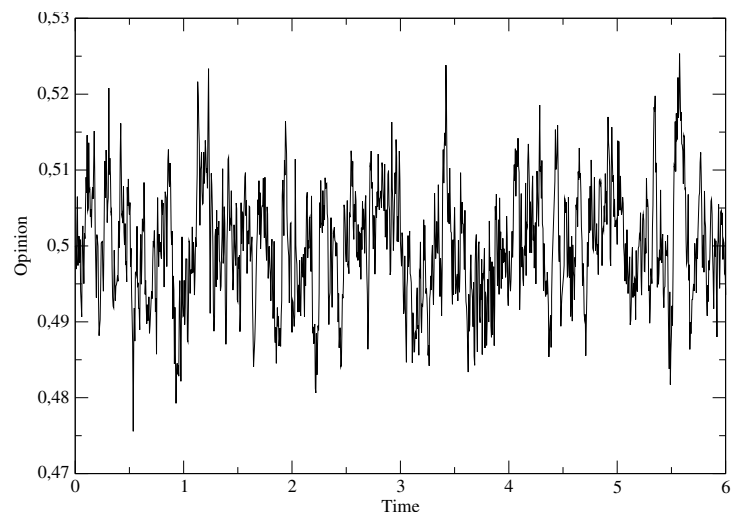

Figure 8. Evolution of $I_{+}$with respect to time (bimodal centred initial datum).

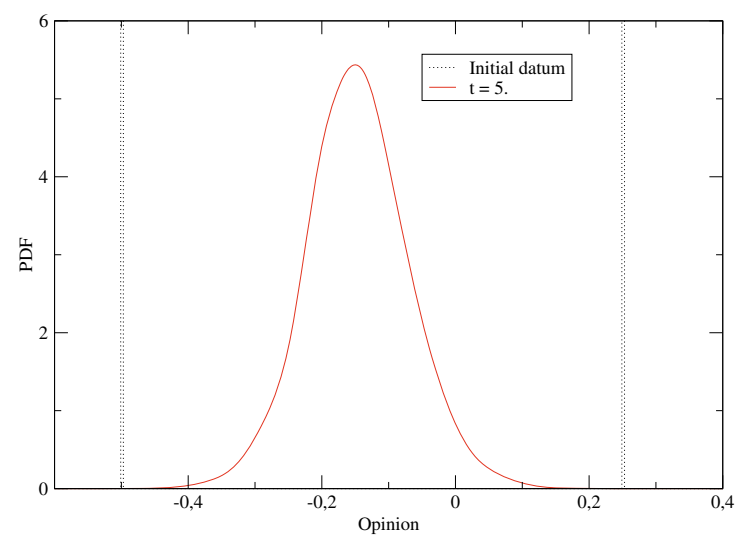

Figure 9. Dirac masses initial datum. 


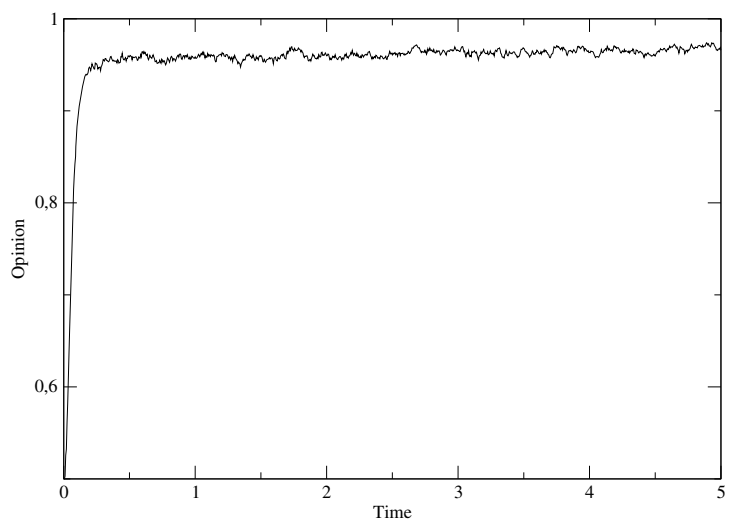

FiguRE 10. Evolution of $I_{-}$with respect to time (Dirac masses initial datum).

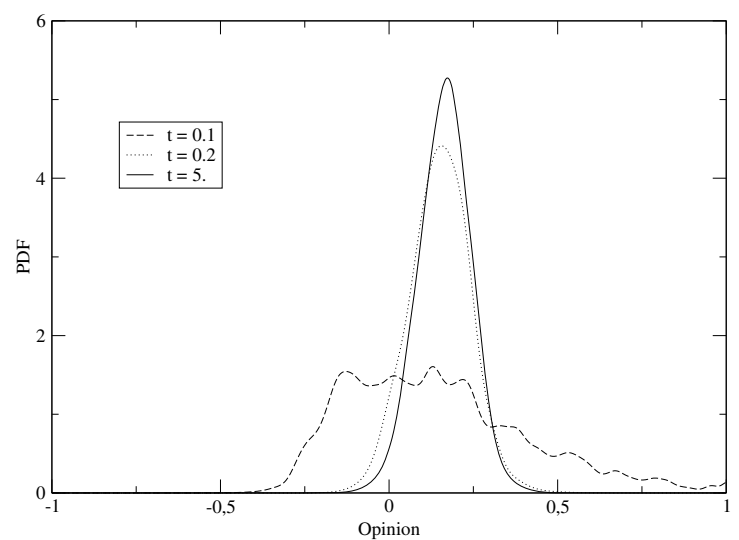

Figure 11. Unbalanced opposite biases case.

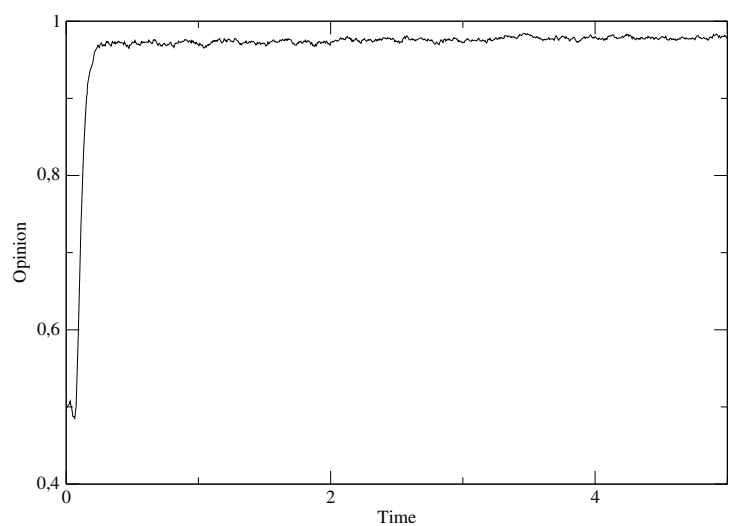

Figure 12. Evolution of $I_{+}$with respect to time (Dirac mass and constant stage initial datum). 
but variations around $50 \%$ are allowed. We find again the conclusion obtained in [9] for a similar initial situation: within a balanced representation group, exchange favours compromise.

\subsection{Two uncentred Dirac masses}

We propose two Dirac masses in -0.5 and 0.25 as the initial datum for $f$, such that the total mass of $f$ remains equal to 1 . The support of the solution significantly changes. Figure 9 shows the situation of $f$ at time $t=5$. Note that the initial datum and the current solution obviously do not share the same scale of density. Indeed, one can only see a small part of the initial datum in Figure 9.

The number of agents who respectively favour positive and negative opinion are initially equal, but the positive opinion is less asserted than the negative one. Figure 10 shows that very fast, the negative opinion becomes hugely majority, but not $100 \%$.

\subsection{Two unbalanced opposite biases case}

We finally consider another case very similar to one studied in [9]. The initial datum $f^{\text {in }}$ equals 1 between -1 and -0.5 , a Dirac mass in 1 , and 0 elsewhere, such that $\left\|f^{\text {in }}\right\|_{L^{1}}=1$. Figure 11 shows three profiles of $f$ with respect to time. It seems to reach an asymptotic equilibrium quite fast.

The profile we obtain for $I_{+}$(see Fig. 12) is very close to the one in Figure 10. The conclusion we can here draw is again the same as in [9]: within an unbalanced representation group, exchange favours the initially strongest representation. Indeed, the positive opinion is hugely majority.

Acknowledgements. This work has been partially supported by the Franco-Italian bilateral Galileo program. We are grateful to Laurent Desvillettes, François Golse, Nicolas Seguin and Giuseppe Toscani for fruitful discussions and comments. F. Salvarani also acknowledges the Laboratoire Jacques-Louis Lions of the Université Pierre et Marie Curie for its hospitality.

\section{REFERENCES}

[1] G. Aletti, G. Naldi and G. Toscani, First-order continuous models of opinion formation. SIAM J. Appl. Math. 67 (2007) 837-853 (electronic).

[2] E. Ben-Naim, Opinion dynamics: rise and fall of political parties. Europhys. Lett. 69 (2005) 671-677.

[3] G.A. Bird, Molecular gas dynamics and the direct simulation of gas flows, Oxford Engineering Science Series 42. The Clarendon Press, Oxford University Press, New York (1995). Corrected reprint of the 1994 original, Oxford Science Publications.

[4] M. Campiti, G. Metafune and D. Pallara, Degenerate self-adjoint evolution equations on the unit interval. Semigroup Forum 57 (1998) 1-36.

[5] J.A. Carrillo, S. Cordier and G. Toscani, Over-populated tails for conservative in the mean, inelastic Maxwell models. Discrete Contin. Dyn. Syst. A (to appear). Available at http://hal.archives-ouvertes.fr/hal-00206273/fr/.

[6] S. Cordier, L. Pareschi and G. Toscani, On a kinetic model for a simple market economy. J. Stat. Phys. 120 (2005) $253-277$.

[7] G. Deffuant, D. Neau, F. Amblard and G. Weisbuch, Mixing beliefs among interacting agents. Adv. Complex Systems 3 (2000) $87-98$.

[8] M.R. Feix, D. Lepelley, V. Merlin and J.-L. Rouet, The probability of conflicts in a U.S. presidential type election. Econom. Theory 23 (2004) 227-257.

[9] S. Galam, Rational group decision making: A random field Ising model at $t=0$. Phys. A 238 (1997) 66-80.

[10] S. Galam, Contrarian deterministic effects on opinion dynamics: the hung elections scenario. Phys. A 333 (2004) 453-460.

[11] S. Galam, Heterogeneous beliefs, segregation, and extremism in the making of public opinions. Phys. Rev. E 71 (2005) 046123.

[12] S. Galam and S. Moscovici, Towards a theory of collective phenomena: consensus and attitude changes in groups. Eur. J. Soc. Psychol. 21 (1991) 49-74.

[13] S. Galam and J.-D. Zucker, From individual choice to group decision-making. Phys. A 287 (2000) 644-659.

[14] S. Galam, Y. Gefen and Y. Shapir, Sociophysics: A new approach of sociological collective behaviour. I. Mean-behaviour description of a strike. J. Math. Sociol. 9 (1982) 1-23.

[15] R. Hegselmann and U. Krause, Opinion dynamics and bounded confidence: models, analysis and simulation. J. Artif. Soc. Soc. Sim. 5 (2002).

[16] F. Slanina, Inelastically scattering particles and wealth distribution in an open economy. Phys. Rev. E 69 (2004) 046102.

[17] F. Slanina and H. Lavička, Analytical results for the Sznajd model of opinion formation. Eur. Phys. J. B 35 (2003) $279-288$.

[18] K. Sznajd-Weron and J. Sznajd, Opinion evolution in closed community. Int. J. Mod. Phys. C 11 (2000) $1157-1166$.

[19] G. Toscani, Kinetic models of opinion formation. Commun. Math. Sci. 4 (2006) 481-496. 